\title{
CARACTERIZACIÓN MORFOLÓGICA Y GENÉTICA DE POBLACIONES DEL GÉNERO PROSOPIS DEL CHACO SEMIÁRIDO DEL NORTE DE CÓRDOBA Y SUR DE SANTIAGO DEL ESTERO
}

\author{
Joseau, M.J." ${ }^{11}$ Verga, A. ${ }^{22}$; Díaz, M del P. ${ }^{33}$ y N. Julio
}

\section{RESUMEN}

De los extensos bosques de Prosopis presentes en Argentina sólo quedan relictos y su explotación irracional genera un empobrecimiento genético, con alarmante pérdida de la biodiversidad. Uno de los relictos importantes lo constituye el corredor que une el Chaco semiárido con el árido, ubicado entre sierras y salinas al norte de Córdoba y sur de Santiago del Estero. En esta región se ponen en contacto cuatro de las especies arbóreas más importantes del género Prosopis ( $P$. chilensis, $P$. flexuosa, $P$. alba y $P$. nigra). Debido a que entre estas especies se forman hibridos fértiles, este corredor posee una enorme diversidad.

El objetivo de este trabajo consistió en caracterizar morfológica y genéticamente, la variación existente en poblaciones del género Prosopis para esta región. Se realizó análisis morfológico tanto en las plantas madres como en la descendencia. En las primeras se identificó la formación de cinco grupos morfológicos que, desde el punto de la taxonomia clásica, habian sido clasificados en 16 taxones. Sólo los grupos 3 y 4 presentaron caracteristicas intermedias. Se analizó la base genética de esos grupos morfológicos mediante marcadores bioquímicos. Se encontró alta correlación entre las matrices de distancia morfológica y genética, principalmente con los sistemas ADH-A y MNR. Los grupos intermedios se diferenciaron del resto también por poseer altos valores de diversidad genética. El estudio morfológico en la descendencia de los grupos morfológicos mostró que el reagrupamiento de los grupos intermedios tuvo una alta tasa de error, reafirmando la condición de grupos intermedios. El análisis de correlación canónica entre las variables morfológicas de las madres y de los hijos reveló que cada grupo morfológico poseía una asociación de variables canónicas propias entre madres e hijos y que la variabilidad observada en el conjunto de las madres y de los hijos sigue un cierto orden.

La gran diversidad morfológica, existente en poblaciones del género Prosopis del Chaco Semiárido del Norte de Córdoba y Sudeste de Santiago del Estero, fue ordenada en 5 grupos morfológicos con una base genética característica para cada grupo. Los estudios morfológicos de la descendencia confirmaron la existencia de los grupos morfológicos-genéticos definidos.

Palabras claves: Caracterización, isoenzimas, Prosopis, grupos morfológicos-genéticos, enjambre hibrido, Chaco árido y semiárido.

\footnotetext{
1 Silvicultura. Facultad de Ciencia Agropecuarias. Universidad Nacional de Córdoba. email: jajoseau@agro.uncor.edu

2IFFIVE-INTA. Córdoba. Argentina

${ }^{3}$ Estadistica y Bioestadistica. Escuela de Nutrición-Facultad de Ciencias Médicas

4 Genética de Poblaciones y Evolución. Facultad de Ciencia Exactas, Fisicas y Naturales.

Universidad Nacional de Córdoba. Argentina.
} 


\section{MORFOLOGIC AND GENETIC CHARACTERIZATION OF PROSOPIS POPULATION IN THE SEMIARID CHACO NORTH OF CORDOBA AND SOUTH OF SANTIAGO DEL ESTERO, ARGENTINA}

\section{SUMMARY}

In Argentina only relicts of the once wide forest of Prosopis remain. The irrational exploitation generates a genetic impoverishment, with an alarming loss of biodiversity. One of the most important relicts is the corridor that unites the semiarid with the arid region located within Sierras and Salinas in the north of Córdoba and south of Santiago del Estero. In this region, four species of Prosopis get in contact ( $P$. chilensis, $P$. flexuosa, $P$. alba y $P$. nigra). Due to the fact that these species make fertile hybrids, this corridor has an enormous diversity.

The objective of this study consisted in the characterization of the genetic and morphological variability present in the Prosopis population in this region. The mother's allowed the differentiation of five morphologic groups that from the point of view of classic taxonomy has been classified in 16 taxa. Only groups 3 y 4 presented intermediate characteristics. It was studied whether those morphologic groups had a genetic basis by means of biochemical markers. High correlation between matrices of morphological and genetic distance was found mainly with $\mathrm{ADH}-\mathrm{A}$ and MNR systems. From the genetic point of view, the intermediate groups also differed from the rest since they had high values of genetic diversity. The morphologic study of the descendants showed that the regrouping of the intermediate groups had a high error rate. This fact reaffirms the condition of intermediate groups. The canonical correlation analysis between the morphologic variables of the mothers and the seedlings revealed that each morphologic group had its own association of canonical variables between mothers and their seedlings, and that the variability observed in the set of the mothers and their seedlings follows a certain order.

The enormous existing morphologic diversity in the Prosopis populations of the semiarid Chaco of the north of Córdoba and south of Santiago del Estero was ordered in five morphologic groups with a characteristic genetic basis for each group. The descendant's morphologic studies confirmed the existence of the defined morphologic-genetic groups.

Key words: Characterization, isozyme, Prosopis, morphologic-genetic groups, hybrid swarms, semi-arid and arid Chaco 


\section{INTRODUCCIÓN}

El problema de la desertificación abarca casi el $40 \%$ de la superficie terrestre. En América Latina y el Caribe este fenómeno cubre un área de más de 600 millones de hectáreas que incluye zonas áridas, semiáridas y subhúmedas (FAO-PNUMA, 1997). En Argentina las especies arbóreas nativas han sufrido y sufren una severa deforestación y en consecuencia, erosión genética. Evidencia de ello es el $60 \%$ de pérdida de bosques nativos en los últimos 100 años (Dirección de Recursos Forestales Nativos, 1992).

Algunas especies arbóreas del género Prosopis son de gran interés económico y ecológico entre los árboles de Argentina. De los extensos bosques de Prosopis presentes en Argentina sólo quedan relictos y su explotación irracional genera un empobrecimiento genético, con alarmante perrdida de la biodiversidad (Julio, 2000).

Uno de los relictos importantes lo constituye el corredor que une el Chaco semiárido con el árido ubicado entre sierras y salinas al norte de Córdoba y sur de Santiago del Estero. En esta región se ponen en contacto cuatro de las especies arbóreas más importantes del género Prosopis ( $P$. chilensis, $P$. flexuosa, $P$. alba y P. nigra). Debido a que entre estas especies se forman hibridos fértiles, este corredor posee una enorme diversidad (Verga, 2000).

Existen enjambres hibridos en muchos grupos de plantas. En las leñosas se han descrito, en los géneros Juniperus, Quercus, Aesculus, Eucalyptus y Opuntia (Grant, 1981) y más recientemente en especies del género Prosopis (Palacios, 1981; Saidman, 1993; Verga, 1995). La taxonomía del género es bastante problemática (Saidman, 1993). Los problemas de la limitación de especies en el género, se dan principalmente en la Sección Algarobia. Al parecer la ausencia clara de barreras de aislamiento reproductivo entre especies (Palacios et al., 1981; Naranjo et al., 1984; Hunziker et al. 1975, 1977, 1986) y la simpatria (Burkart, 1976; Morello et al., 1971; Ferreyra, 2001) han facilitado la hibridación e introgresión y han contribuido a la variabilidad morfológica y genética observada en sus poblaciones (Ferreyra, 2001).

Las especies ligadas por hibridación frecuente u ocasional constituyen un singámeon. Este fue definido por Grant (1981) como un grupo de especies que hibridan y se comportan como una especie biológica, aislada reproductivamente de otros grupos similares. Dada la capacidad de hibridación de algunas especies de la Sección Algarobia ( $P$. alba, $P$. nigra, $P$. chilensis y P. flexuosa) algunos autores (Palacios et al., 1981; Saidman, 1986, Bessega, 1997) han sugerido que las mismas constituirian un singámeon, lo cual dificulta su identificación a campo.

Estudios previos, en la Sección Algarobia, cromatográficos (Solbrig et al, 1977; Palacios y Bravo, 1981; Naranjo y Enus Zelger, 1983, Naranjo et al., 1984), de electroforesis de proteinas seminales (Burghardt y Palacios, 1981 y Burghardt, 1992) y de electroforesis de isoenzimas (Saidman, 1985, 1986, 1988, 1993, Saidman y Vilardi, 1987; Verga 1995) indicaron un bajo grado de diferenciación entre especies, lo cual podria ser explicada por la hibridación frecuente entre ellas en zona de simpatria, favorecida por barreras de aislamiento débiles y por modificaciones del ambiente (Julio, 2000). Sin embargo, estudios taxonómicos morfológicos señalan una gran diferenciación entre las distintas especies (Burkart, 1976; Solbrig et al., 1977, Verga, 1995). 
Esta aparente contradicción, se genera debido a que existen individuos que representan fielmente a las especies mencionadas, las cuales inclusive se diferencian nitidamente, tanto desde el punto de vista morfológico como ecológico. No obstante, cuando se estudian regiones donde estas especies crecen en contacto, la existencia de un número importante de individuos intermedios hace que gran parte de ellos no puedan ser clasificados con claridad en ninguna de las especies presentes. Esto genera a su vez, que cuando se realizan estudios sobre una determinada especie, el resultado dependerá de cuál ha sido el criterio para seleccionar los individuos que representarán la especie en cuestión. Si se incluyen únicamente los que responden estrictamente a las caracteristicas de las especies puras, la diferenciación entre éstas será importante y la variación intraespecifica tanto genética como morfológica será menor. A medida que se van incorporando individuos intermedios, que responden parcialmente a las características de la especie, ésta se va desdibujando con respecto a las otras afines, su diferenciación disminuye y su diversidad aumenta (Verga, com.pers.).

La hibridación del ambiente que ocurre debido a la actividad humana, como la construcción de caminos, la agricultura, el riego y la explotación forestal (Anderson, 1949, Grant, 1981, Palacios et al., 1981) es una de las condiciones que favorecen el establecimiento de los hibridos (Grant, 1981, Palacios et al., 1981).

\section{OBJETIVO}

El objetivo de este trabajo consistió en caracterizar morfológica y genéticamente, la variación existente en poblaciones del género Prosopis para esta región.

\section{MATERIALES Y MÉTODOS}

El presente trabajo de investigación se efectuó con material cosechado de árboles de Prosopis chilensis, P. alba, P. flexuosa, P. nigra e individuos intermedios, posibles híbridos entre estas cuatro especies, en la región del Chaco Semiárido del norte de Córdoba y el sur de Santiago del Estero.

La metodología para ordenar el aparente continuo consistió en diferenciar los grupos morfológicos en el área de contacto a través del análisis morfológico realizado a las plantas madres y a los descendientes. Se efectuó un análisis genético para comprender si el ordenamiento morfológico obtenido y confirmado mediante análisis discriminante se correspondía con el genético. Para ello se estableció la estructura genética de los grupos morfológicos, el de las poblaciones y las correlaciones existente entre ambas distancias (morfológicas y genéticas).

\section{Análisis Morfológico de las Plantas Madres}

Se tomó muestras de 10 hojas y 10 frutos de los 79 ejemplares muestreados en cuatro zonas (poblaciones) seleccionadas en base a gradientes de temperatura y precipitaciones en la región del Chaco Semiárido del norte de Córdoba y el sur de Santiago del Estero (Cuadro $N^{\circ}$ 1). Se preparó herbarios que fueron remitidos al Laboratorio de Sistemática y Plantas Vasculares de la Universidad de Buenos Aires para la identificación botánica mediante 
taxonomia clásica. Por otra parte se efectuó un análisis de taxonomia numerica con las muestras tomadas a campo de frutos y hojas. Para la construcción de los grupos morfológicos sobre un total de 19 variables se estableció la distancia taxonómica y se construyó un dendrograma.

\section{Análisis Morfológico de los Descendientes}

Se efectuó mediciones de caracteres de hojas y de crecimiento, como la altura a cuatro edades del plantin, la forma del tallo principal y el grado de presencia de espinas a los 180 dias del plantín. Con los datos obtenidos se realizó un análisis discriminante cuadrático.

\section{Cuadro $N^{\circ} 1$}

\section{CANTIDAD Y LOCALIZACIÓN DE LOS ALGARROBOS COSECHADOS}

\begin{tabular}{|l|c|c|c|c|c|c|c|}
\hline Zona & Latitud S & Longitud W & $\begin{array}{c}\text { Altitud } \\
(\mathrm{msnm})\end{array}$ & Cantidad & $\begin{array}{c}\text { pp media } \\
\text { anual } \\
(\mathrm{mm})\end{array}$ & $\begin{array}{c}\text { Temperatura } \\
\text { media mes } \\
\left({ }^{\circ} \mathrm{C}\right)\end{array}$ \\
\hline & & & & & & $\begin{array}{c}\text { Más } \\
\text { cálido }\end{array}$ & $\begin{array}{c}\text { Más } \\
\text { frio }\end{array}$ \\
\hline 1.El Jume-Los Telares & $28^{\circ} 59^{\circ}-29^{\circ} 23^{\circ}$ & $63^{\circ} 26^{\circ}-63^{\circ} 41^{\circ}$ & $108-480$ & 27 & $597-600$ & 26,9 & 11,5 \\
\hline $\begin{array}{l}\text { 2 L. V Mansilla San José } \\
\text { de La Salinas }\end{array}$ & $29^{\circ} 47^{\circ}-30^{\circ} 08^{\prime}$ & $63^{\circ} 42^{\circ}-64^{\circ} 31^{\circ}$ & $191-193$ & 15 & $394-453$ & 27,7 & 12,5 \\
\hline 3.Quilino-Huascha & $30^{\circ} 12^{\circ}-30^{\circ} 36^{\circ}$ & $64^{\circ} 24^{\circ}-64^{\circ} 46^{\circ}$ & $393-689$ & 23 & $445-560$ & 26,2 & 10,8 \\
\hline 4.Cruz del Eje- El Carrizal & $29^{\circ} 38^{\circ}-30^{\circ} 45^{\circ}$ & $64^{\circ} 38^{\circ}-65^{\circ} 14^{\circ}$ & $275-600$ & 19 & $379-481$ & 27,6 & 11,5 \\
\hline
\end{tabular}

(Fuente: De Fina, 1976)

\section{Relaciones Morfológicas entre las Plantas Madres y la Descendencia.}

Se utilizó un análisis de correlaciones canónicas (ACC) entre el grupo de variables morfológicas de las madres y el grupo de variables de sus descendencias para cada grupo morfológico y se verificó su significancia estadistica.

\section{Análisis Genético}

Se analizó 6 sistemas isoenzimáticos: Alcohol deshidrogenada (ADH), glutamato oxalacetato transaminasa (GOT), 6-fosfogluconato deshidrogenasa (6PGDH), menadione reductasa (MNR), fosfoglucosaisomerasa (PGI) y shikimico deshidrogenasa (SKDH). La metodologia aplicada fue la descripta por Verga (1995).Se analizaron entre 10 a 15 semillas por árbol como minimo.

En la comparación de las estructuras genéticas de los grupos morfológicos se utilizó la distancia genética $d_{o}$ (Gregorius, 1974) que permite realizar comparaciones con la distancia morfológica $d_{m}$. La variabilidad genética de los grupos morfológicos se cuantificó por medio de los indices: los índices de diversidad $v$ (número efectivo de alelos) y de diferenciación total de

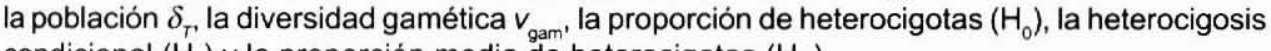
condicional $\left(\mathrm{H}_{c}\right)$ y la proporción media de heterocigotas $\left(\mathrm{H}_{\mathrm{m}}\right)$. 


\section{RESULTADOS Y DISCUSIÓN}

\section{Identificación Botánica de las Plantas Madres} clásica.

En la el Cuadro $N^{\circ} 2$ se presenta los 16 taxones identificados mediante la taxonomía

\section{Clasificación Mediante Taxonomia Numérica}

A partir de la matriz de distancia $d_{m}$ se construyó el dendrograma de la Figura $N^{\circ} 1$. En dicha figura se observa que a un nivel del $78 \%$ se diferenciaron dos grandes grupos. Desde el árbol 1 al 52, que correspondería al grupo de los "blancos" y desde el 3 al 85 que representarian a los «negros». Dentro de cada uno de estos dos grandes grupos se forman claramente, sub-grupos que, en algunos casos, podrían corresponder a diferencias entre especies o a la presencia de individuos intermedios. A un nivel del $70 \%$ se observa la formación de 5 grupos (Figura $\mathrm{N}^{\circ} 1$ ).

Si se compara estos grupos con las determinaciones hechas a través de la taxonomía clásica, el grupo 1 está formado por individuos clasificados como $P$. alba, $P$. chilensis, $P$. chilensis con foliolos menores e híbridos entre las dos especies mencionadas; al 2 lo forman individuos clasificados como $P$. chilensis principalmente y el grupo 3 se corresponde con $P$. chilensis y tres individuos híbridos entre esta especie y $P$. alba. El grupo 4 es un grupo totalmente intermedio formado por distintos tipos de híbridos (algarrobos blancos por negros o viceversa) y el 5 está integrado principalmente por $P$. nigra.

\section{Cuadro $\mathrm{N}^{\circ} 2$}

\section{IDENTIFICACIÓN DE LAS PLANTAS MADRES POR MEDIO DE LA TAXONOMIA CLÁSICA}

\begin{tabular}{|c|c|c|c|}
\hline Especie & Simbolo & Número de la Planta Madre & Total \\
\hline P. alba & $\mathrm{Pa}$ & $11-12-18-20-26-84$ & 6 \\
\hline P. alba (foliolos algo menores) & $\mathrm{Pa}_{\mathrm{h}}$ & 23 & 1 \\
\hline P. alba $x$ ? & Pax? & $2-31-39-52$ & 4 \\
\hline P. alba $\times$ P.nigra & Paxn & 22 & 1 \\
\hline P. alba o P.chilensis $\times$ P. flexuosa & Paxf & 41 & 1 \\
\hline P.chilensis $\times$ P alba & Pcxa & $1-4-5-8-24-36$ & 6 \\
\hline P.chilensis & $\mathrm{Pc}$ & $19-35-37-43-44-46-50-51-55-56-59-60-61-62-63-64-65-66-69-70-74-77-79-80-82$ & 25 \\
\hline P.chilensis (foliolos algo menores) & $\mathrm{PC}_{\mathrm{ln}}$ & $10-17-32-33-38-42-45-57-78$ & 9 \\
\hline P.chilensis $x$ ? & Pcx? & $27-40$ & 2 \\
\hline P.chilensis $\times P$, flexuosa & Pcxt & $81-83$ & 2 \\
\hline Pchilensis $\times$ P. flexuosa o P.nigra & Pcxfon & $68-75-76$ & 3 \\
\hline Pchilensis $\times$ P ruscifolia & Pcxr & 21 & 1 \\
\hline P. ruscifolia & $\mathrm{Pr}$ & 25 & 1 \\
\hline P.flexuosa o P.flexuosa $\times$ P.alba & Pfo Pfxa & $3-6$ & 2 \\
\hline P.flexuosa o P.nigra $\times$ P.chilensis & Pfxc & 53 & 1 \\
\hline P.nigra & Pn & $7-9-13-14-15-16-28-30-34-48-49-54-58-67-71-72-73-85$ & 18 \\
\hline Sin identificar & S/ident. & $29-47$ & 2 \\
\hline Total de taxones & 16 & & 85 \\
\hline
\end{tabular}




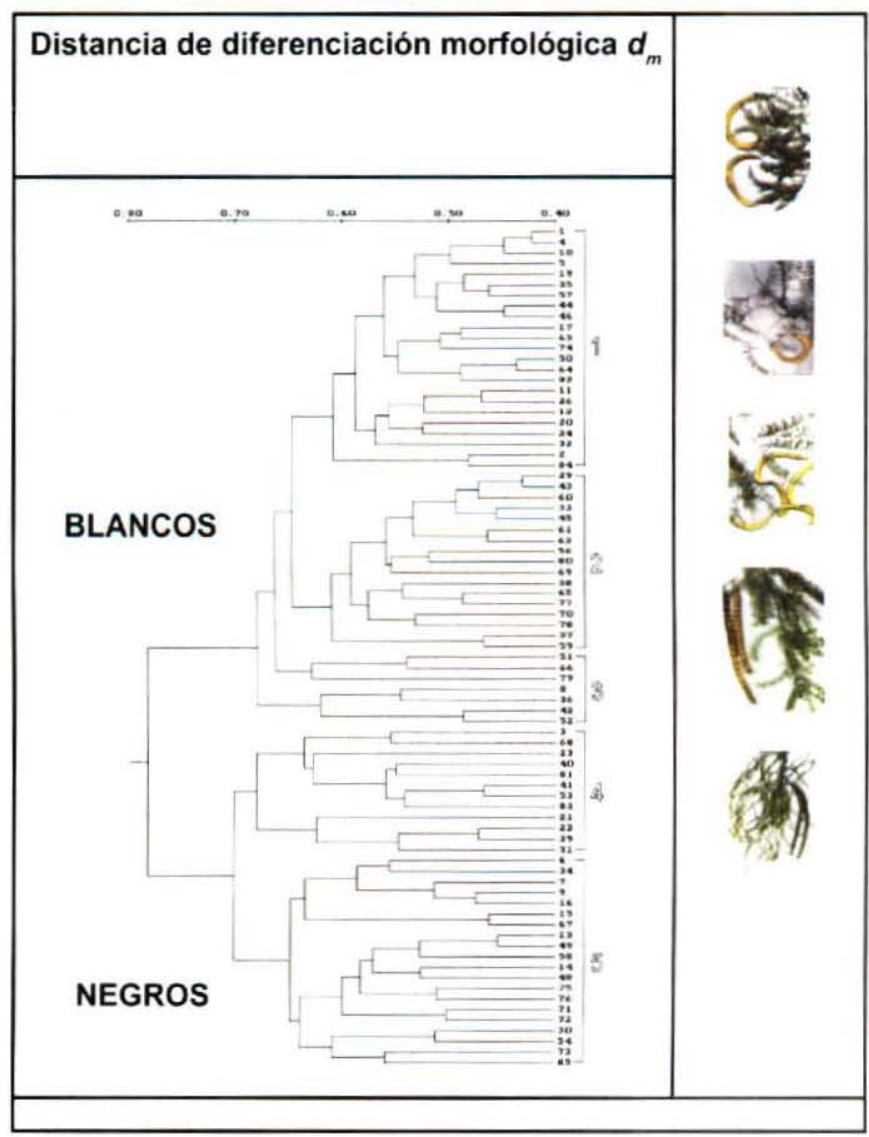

FIGURA $N^{\circ} 1$

DENDROGRAMA OBTENIDO CON EL MÉTODO UPGMA,

BASADO EN LA DISTANCIA MORFOLİGICA ENTRE LOS INDIVIDUOS REPRESENTADOS POR TODOS LOS CARACTERES MORFOLOGICOS DE LAS PLANTAS MADRES

\section{Análisis Discriminante de las Madres}

El empleo de la taxonomia numérica constituye una herramienta fundamental para el análisis morfológico y la correcta identificación de los materiales utilizados en este estudio. Asi fueron 9 los caracteres, de un total de 19, que contribuyeron de manera significativa a la construcción de los grupos morfológicos de las madres. Los caracteres de fruto de mayor correlación con las funciones discriminantes estimadas fueron el color y el ancho de fruto. Los caracteres de fruto y rama son los que han tenido mayor participación en la formación de los grupos. En cuanto a los caracteres de hoja, sólo participaron la relación longitud/ancho de foliólulo y el número de pinas por hoja. 
Verga (1995) encontró que los caracteres relación espesor/ancho de fruto, relación longitud/ancho de foliólulo y la longitud del foliólulo fueron importantes para la diferenciación de tres grupos morfológicos ( $P$. flexuosa, $P$. chilensis y sus híbridos) en el Chaco árido.

Asimismo, Mantován (2000) encontró que la diferenciación morfológica entre poblaciones de Prosopis flexuosa en la provincia fitogeográfica del Monte fue posible gracias al gran peso que presentaron variables como la longitud de foliólulo y la relación entre la longitud de la pina y el ancho del foliólulo.

Por otra parte, Burghardt et al. (2000) determinaron que variables que constituyen magnitudes absolutas tales como largo y ancho de pina y longitud de foliolo, evaluadas a través de análisis de componentes principales, fueron adecuadas para diferenciar plántulas de 8 especies de Prosopis, cultivadas en condiciones uniformes.

Del análisis morfológico de las madres surge que fue posible agrupar el aparente continuo en cinco grupos morfológicos con un nivel de error del $24 \%$. Este error fue atribuible, principalmente a la variabilidad en el grupo 3 y en menor medida en el grupo 4 , ambos grupos catalogados como intermedios. Los grupos 1 y 2 fueron los grupos más homogéneos, dado que el porcentaje de error fue muy bajo (4 a 5,6\%), seguido por el grupo $5(15 \%)$.

\section{-La Clasificación}

Los grupos morfológicos que surgen del ordenamiento de las madres, (determinados mediante taxonomía numérica y confirmados, con un cierto error de mala clasificación, mediante análisis discriminante, tanto en las madres como en los hijos) son grupos homogéneos y se considera que cada uno de ellos posee una única identidad. Esta única identidad no concuerda con la clasificación realizada desde la taxonomia clásica.

La taxonomia clásica determinó que en este estudio existen 16 taxones, mientras que el estudio morfológico los reduce a 5 grupos morfológicos (Joseau et al., 2004, Joseau y Castro Schüle, 2005).

\section{- De los Descendientes}

Se estudió si la descendencia obedecia a las agrupaciones obtenidas en las plantas madres mediante análisis discriminante. Dado que no se contaba con igual cantidad de datos, se hizo dos evaluaciones: 1.- para caracteres de hojas semejantes a las medidas en las plantas madres y 2.- para caracteres registrados directamente en el vivero como altura en diferentes fechas, forma del tallo y grado de presencia de espinas.

\section{- Análisis Discriminante}

Tanto en los caracteres del punto 1 como del 2, la tasa de error total de este análisis fue muy alta $(0,514$ y 0,595 respectivamente) mostrando que no es posible agrupar de la 
misma manera a la descendencia que a las plantas madres. Sólo los descendientes del grupo 5 se reagrupan en un $85 \%$, para el primer conjunto de variables y en un $81 \%$ para el segundo conjunto constituyéndose asi en el grupo más estable en la descendencia.

Para resumir, es licito aclarar, que es difícil agrupar los descendientes bajo los agrupamiento de las plantas madres considerando estos caracteres, puesto que existe una alta tasa de error. El error para las variables morfológicas de hojas es aportado en mayor porcentaje por los grupos 1,3 y 4 , mientras que para las variables de crecimiento los grupos que más error aportan son 2,3 y 4 .

En este estudio, para el análisis morfológico de la descendencia, fueron necesarias 8 de las 12 variables evaluadas a nivel de hojas y 5 de las 6 variables registradas en vivero, para lograr una diferenciación adecuada. La longitud del foliólulo conjuntamente con el ancho y el área de los mismos constituyeron las variables de mayor peso en el análisis discriminante.

\section{Distribución de los Grupos Morfológicos por Zonas}

Observando la distribución de los grupos morfológicos genéticos por zonas, la zona 1 (Santiago del Estero) es la que más se diferencia del resto, dado que el grupo morfológico 2 (P. chilensis) está ausente. Las restantes zonas (Córdoba) poseen los 5 grupos morfológicos pero en diferentes proporciones (Figura $\mathrm{N}^{\circ} 2$ ).

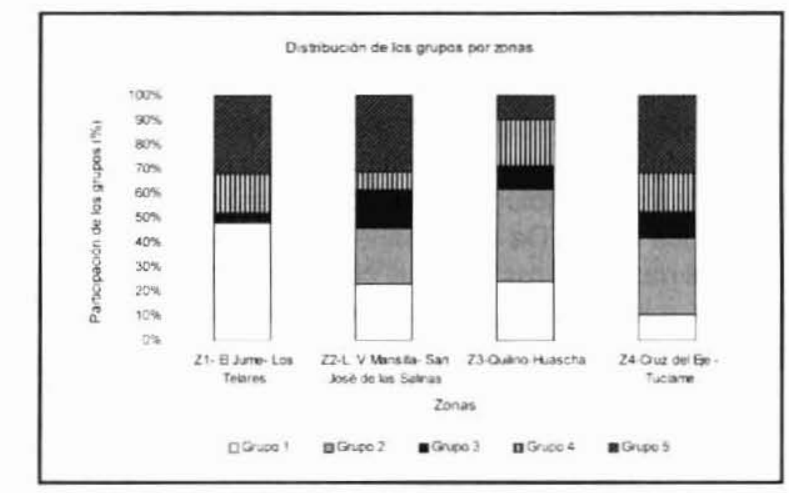

Figura $\mathrm{N}^{\circ} 2$

DISTRIBUCIÓN DE LOS GRUPOS MORFOLÓGICOS POR ZONAS

\section{Estudio de la Asociación entre Caracteres de los Árboles Semilleros y sus Descendencias}

La relación existente entre los grupos morfológicos de los árboles cosechados y de la descendencia, evaluada a través del análisis de correlaciones canónicas, se efectuó entre el grupo de variables morfológicas de las madres (exceptuando tipo de rama) y el grupo de variables de los hijos para cada grupo morfológico. Si bien los datos se presentan en forma conjunta, cada grupo morfológico tuvo un análisis de correlación canónica independiente de los restantes grupos. 
La mayoria de los grupos morfológicos necesitaron dos pares de variables canónicas (L) para establecer asociaciones entre los grupos de variables de las madres y de los hijos, con excepción del grupo 3 en el cual esta asociación quedó bien definida con sólo una correlación canónica (Tabla 3 ).

Si bien para todos los grupos la correlación canónica es altamente significativa, se destacan los grupos 3 y 4 presentando los mayores valores de $R^{2}$ (Cuadro $N^{\circ} 3$ ). Esta correlación indica que la variabilidad encontrada en las madres está fuertemente asociada con la variabilidad encontrada en los hijos, en otras palabras, la variabilidad observada en ambos conjuntos sigue un cierto orden o relación entre pares de conjuntos de variables.

\section{Cuadro $\mathrm{N}^{\circ} 3$}

COEFICIENTES DE CORRELACIÓN CANÓNICA (R), LA PROPORCIÓN DE LA VARIANZA TOTAL EXPLICADA POR CADA PAR DE VARIABLES CANONICAS $\left(\mathbf{R}^{2}\right)$

Y NIVELES DE PROBABILIDAD ASOCIADOS (P-VALOR) PARA CADA PAR DE VARIABLES CANÓNICAS Y PARA CADA GRUPO MORFOLÓGICO

\begin{tabular}{|c|c|c|c|c|c|c|c|c|c|}
\hline Grupo & \multicolumn{2}{|c|}{1} & \multicolumn{2}{c|}{2} & 3 & \multicolumn{2}{c|}{4} & \multicolumn{2}{c|}{5} \\
\hline $\mathrm{L}$ & 1 & 2 & 1 & 2 & 1 & 1 & 2 & 1 & 2 \\
\hline $\mathrm{R}$ & 0,72 & 0,55 & 0,68 & 0,67 & 0,94 & 0,82 & 0,68 & 0,75 & 0,60 \\
\hline $\mathrm{R}^{2}$ & 0,51 & 0,30 & 0,46 & 0,45 & 0,89 & 0,67 & 0,46 & 0,57 & 0,37 \\
\hline p-valor $^{*}$ & $1,4.10^{-9}$ & $4.7 .10^{-3}$ & $7,4.10^{-8}$ & $1,6.10^{-4}$ & $5,3.10^{-4}$ & $2,7.10^{-7}$ & $3,7.10^{-3}$ & $5,3.10^{-10}$ & $1,5.10^{-3}$ \\
\hline
\end{tabular}

* $\mathrm{p}$-valor $<0,001$ se considera altamente significativa.

Cabe destacar que las combinaciones lineales con máxima correlación para cada par (L) y para cada grupo morfológico se formaron con diferentes asociaciones de variables, tanto para el primer conjunto (variables morfológicas de las madres) como para el segundo conjunto (variables morfológicas de los hijos). De esta manera, se puede decir, en el conjunto de las madres que para los grupos morfológicos 1 y 2 los caracteres de fruto tuvieron un mayor peso que los caracteres de hojas, mientras que para los restantes grupos la participación de ambos tipos de caracteres fue equitativa.

Si bien no se puede corroborar la formación de los mismos agrupamientos en los hijos, el análisis multivariado de correlación canónica para cada grupo morfológico refleja que los grupos morfológicos que más explican la variabilidad para cada par de ejes canónicos fueron los grupos más variables ( 3 y 4 ) al presentar los mayores valores de $R^{2}$. Estos valores indican que la alta variabilidad encontrada en las madres está fuertemente asociada a la alta variabilidad encontrada en los hijos, en otras palabras, la variabilidad observada en ambos conjuntos sigue una cierta relación lineal, o podria decirse, un orden continuo.

Cada par de ejes canónicos fue diferente para cada grupo morfológico, lo que reafirma cierta identidad de los grupos morfológicos. A este nivel de análisis se destacan variables como las citadas por Burghardt et al. (2000). La longitud de la pina, el número de foliólulos y la longitud de foliólulos en el conjunto de los hijos y para el conjunto de las madres las variables que más se repitieron en las distintas asociaciones fueron el número de artejos, el espesor del fruto, la longitud del foliólulo y la relación entre la longitud y el ancho del foliólulo. 


\section{Análisis Genético}

Los zimogramas de las enzimas analizadas revelaron la existencia de 9 loci con sus respectivos alelos (ADH-A, ADH-B, GOT-A, GOT-B, 6PGDH-A, 6PGDH-B, MNR, PGI y SKDH).

Las frecuencias alélicas fueron calculadas a partir de las observaciones obtenidas en los zimogramas para cada sistema isoenzimático. Los distintos loci y alelos observados en el análisis del material utilizado para este trabajo son designados según la nomenclatura propuesta por Verga (1995).

De los 6 sistemas estudiados, que comprenden nueve loci, se encontró sólo uno fijado (ADH-B). Los loci ADH-A y MNR alcanzaron los mayores grados de la diferenciación total entre los grupos morfológicos $\left(\delta_{\mathrm{ADH}-\mathrm{A}}=0,245, \delta_{\mathrm{MNR}}=0,206\right)$ (Figuras $\mathrm{N}^{\mathrm{os}} 3$ y 4 ).

ADH-A

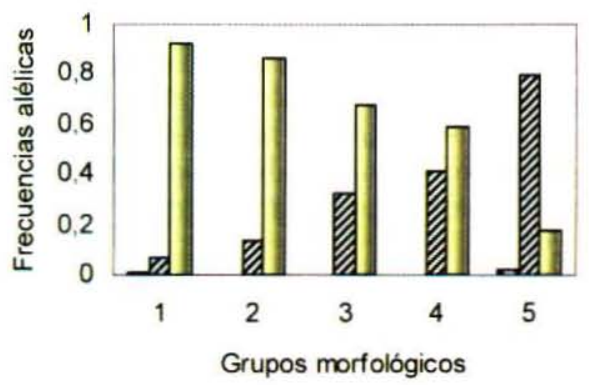

จAlelo 1 Alelo 2 凹 Alelo 3

Figura $\mathrm{N}^{\circ} 3$

FRECUENCIAS ALÉLICAS DE LOS GRUPOS MORFOLÓGICOS PARA ADH-A

La estructura genética de los grupos morfológicos para el locus ADH-A, permitió diferenciar claramente los grupos 1 y 2 del 5 . Por otra parte los grupos 1 y 2 se diferencian entre si en forma cualitativa puesto que el 1 posee al alelo $A D H-A$, en baja frecuencia, mientras que el grupo 2 no lo posee. Los grupos 3 y 4 aparecen con frecuencias intermedias de los alelos 2 y 3 .

Verga $(1995,2000$ a) pudo diferenciar poblaciones de $P$. chilensis, $P$. flexuosa y un grupo con características intermedias en el Chaco Árido, dado que el alelo ADH-A se encontraba fijado en $P$. chilensis, casi ausente en la otra especie y en los híbridos putativos presentaba una frecuencia intermedia.

En este estudio si bien el alelo $\mathrm{ADH}-\mathrm{A}_{3}$ no aparece fijado, en las poblaciones del grupo 2 ( $P$. chilensis) se encontró en alta frecuencia (superior al $80 \%$ ) de igual manera que en el grupo 1 ( $P$. alba). Por otra parte, el alelo $\mathrm{ADH}-\mathrm{A}_{2}$ mostró un comportamiento similar al encontrado 
por Verga (1995, 2000 a), Verga et al. (2004), Verga y Gregorius (no publicado) para P. flexuosa, que es un algarrobo negro, puesto que tuvo una alta frecuencia en el grupo 5 conformado en este análisis por otro algarrobo negro ( $P$. nigra).

Saidman (1985, 1986, 1993), Saidman \& Vilardi (1987, 1993), Saidman et al. (1997 a, 1998), Julio (2000) y Ferreyra (2000) estudiaron este locus y no encontraron ningún patrón que confirme una determinación morfológica, posiblemente porque estos estudios sólo fueron de indole genético, clasificando el material únicamente mediante taxonomia clásica. Es de destacar en este punto que la difícil clasificación de las especies, por el método clásico, puede inducir a confusiones en cuanto al material que se está utilizando en el análisis genético.

La inclusión de material de origen hibrido dentro del estudio de especies puras puede asi llevar a conclusiones contradictorias. Si la cantidad de individuos intermedios incluidos en un estudio es lo suficientemente importante, es muy probable que las diferencias genéticas entre especies disminuyan considerablemente y que el grado de variación genética de ellas sea sobredimensionado.

También la confusión que incorporaria una clasificación no adecuada del material de trabajo podria llevar a errores en la determinación del grado de diferenciación entre poblaciones de una misma especie, ya que el resultado no dependerá únicamente de cambios en las frecuencias genéticas debido a procesos evolutivos, sino además del grado de "contaminación" del material de estudio con individuos de origen hibrido.

El locus MNR presentó cinco alelos para el grupo morfológico 1, el 5 en muy baja frecuencia y el alelo 2 con frecuencia intermedia de 0,530 . Por otra parte se observa que el grupo morfológico 2 se diferencia del resto por presentar frecuencias del alelo 3 superior al 2 . Al grupo morfológico 3 se lo puede individualizar pues presenta frecuencias del alelo 1 superior al resto de los grupos $(0,162)$. Si bien los grupos 4 y 5 muestran una frecuencia alta del alelo 2 , el grupo 4 presenta valores menores, casi un $17 \%$ menos, que el 5 (Figura $\mathrm{N}^{\circ} 4$ ).

\section{MNR}

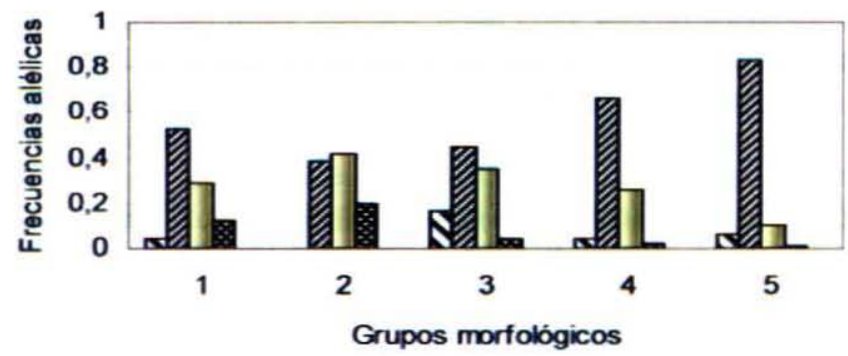

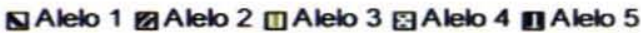

Figura $\mathrm{N}^{\circ} 4$

FRECUENCIAS ALÉLICAS DE LOS GRUPOS MORFOLÓGICOS PARA MNR 
El locus MNR mostró una estructura diferencial para cada grupo morfológico, y si se compara con los resultados obtenidos por Verga (1995) es posible identificar la estructura obtenida para $P$. chilensis como similar a la encontrada para el grupo 2 de este estudio. No se encontró otras investigaciones que utilicen está isoenzima en Prosopis razón por lo cual resulta difícil su comparación.

\section{Medidas de Variación Genética en los Grupos Morfológicos}

En el Cuadro $\mathrm{N}^{\circ} 4$ aparece la diversidad genética de los grupos para los loci ADH-A y MNR. Los grupos 3 y 4 presentan los mayores valores en los índices de diversidad $v$ (número efectivo de alelos) y de diferenciación total de la población $\mathrm{d}_{\tau}$ para ADH-A. Para el locus MNR la mayor diversidad estaria concentrada en los grupos morfológicos 3,2 y 1 en orden decreciente de importancia (Cuadro $\mathrm{N}^{\circ} 4$ ).

\section{Cuadro $\mathrm{N}^{\circ} 4$}

DIVERSIDAD $v$ Y $\delta_{T}$ DE LOS GRUPOS MORFOLOGICOS PARA LOS LOCI ADH-A Y MNR

\begin{tabular}{|l|c|c|c|c|c|}
\hline ADH-A & Grupo 1 & Grupo 2 & Grupo 3 & Grupo 4 & Grupo 5 \\
\hline Diversidad $v$ & 1,173 & 1,317 & 1,772 & 1,955 & 1,497 \\
\hline$\delta_{\boldsymbol{T}}$ & 0,148 & 0,242 & 0,437 & 0,49 & 0,333 \\
\hline MNR & Grupo 1 & Grupo 2 & Grupo 3 & Grupo 4 & Grupo 5 \\
\hline Diversidad $v$ & 2,587 & 2,777 & 2,860 & 1,918 & 1,418 \\
\hline$\delta_{\boldsymbol{T}}$ & 0,615 & 0,642 & 0,652 & 0,480 & 0,296 \\
\hline Pool de genes & Grupo 1 & Grupo 2 & Grupo 3 & Grupo 4 & Grupo 5 \\
\hline Diversidad $v$ & 1,610 & 1,705 & 1,864 & 1,738 & 1,794 \\
\hline$\delta_{\boldsymbol{T}}$ & 0,380 & 0,416 & 0,466 & 0,426 & 0,424 \\
\hline Diversidad $v_{\text {gam }}$ & 118,530 & 203,844 & 449,818 & 223,667 & 208,967 \\
\hline
\end{tabular}

Cuanto más cerca de 1 se encuentra el valor de $\delta_{T}$ más se diferencian los tipos genéticos que conforman cada grupo morfológico. Asi los grupos 3 y 4 son los que contienen mayor número de individuos en la muestra que no corresponden al mismo tipo genético.

El locus ADH-A puede ser consiedrado como marcador genético para diferenciar los grupos morfológicos. La tendencia que se observa en las distancias genéticas $d_{0}$ para ADH-A es casi la misma que se presenta en el pool de genes, de tal manera que la correlación fue significativa $(\mathrm{P}<0,05)$ entre las matrices de distancias genéticas $d_{o}$ para $\mathrm{ADH}-\mathrm{A}$ y el pool de genes con un $R^{2}=0,89$.

Las distancias genéticas entre los grupos morfológicos de la enzima MNR sigue aproximadamente el mismo patrón que la enzima $\mathrm{ADH}-\mathrm{A}$ y el pool génico, aunque con valores menores $(P<0,05)$ de $R^{2}(0,4356$ con $A D H-A$ y 0,4489 con el pool de genes).

Las medidas de variación de las muestras del pool génico estarian indicando que la mayor diversidad se encontraría en el grupo 3 y 4 (Cuadro $N^{\circ} 4$ ). Se sabe que $v_{\text {gam }}$ mide el 
potencial de una población para producir gametas genéticamente distintas. Asi se ve en el cuadro mencionado que el grupo 3 duplica el valor registrado en el grupo 4 que es el inmediato inferior, mientras que el grupo 1 presenta la menor diversidad.

Los loci ADH-A y MNR permitirian la diferenciación de los grupos morfológicos, siendo el locus ADH-A el que posee una tendencia más parecida a la que presenta el pool de genes en cuanto a las distancias $d_{0}$ entre los grupos morfológicos.

En el Cuadro $\mathrm{N}^{\circ} 5$ se indica los valores alcanzados por los siguientes indices para cada grupo morfológico: Proporción de heterocigotas $\left(\mathrm{H}_{0}\right)$, Heterocigosis condicional $\left(\mathrm{H}_{\mathrm{c}}\right)$ y proporción media de heterocigotas $\left(H_{m}\right)$. Se desprende de estos datos que los grupos 3 y 4 , formados por individuos de características morfológicamente intermedias, son los que mayores valores presentan en estos índices.

Para ADH-A, se observa la misma tendencia, siendo los valores más pequeños los presentes en los grupos 1, 2 (grupos de blancos) y 5 (grupo de negros).

Las especies de Algarobia muestran una alta tasa de variabilidad genética dentro de poblaciones, medidas en términos de heterocigosis $(\bar{H}=0,21)$ que aquellas especies de la sección Strombocarpa $(\bar{H}=0,06)$ (Saidman et al., 2000).

Las especies de Prosopis de este estudio pertenecen a la sección Algarobia, son especies leñosas de larga vida con amplio rango geográfico, sistema de cruzamiento abierto principalmente, dispersan la semilla por ingestión animal y presentan altos valores de diversidad que varian según el grupo morfológico

Coincidentemente, Verga (1995) encontró en estudios isoenzimáticos para 9 loci en poblaciones de Prosopis del Chaco Árido que los mayores niveles de diversidad alélica $\delta \tau$ se encontraban en el grupo de características intermedias tanto para el locus ADH-A como para el pool de genes.

En este estudio los valores de heterocigosis tuvieron un rango entre 0,22 a 0,34 . Así se tiene que la proporción media de heterocigotas $(\bar{H})$ fue superior para los grupos 3 y 4 ( $\bar{H}=0,34$ y $\bar{H}=0,32$ ), que estaban conformados por individuos clasificados mediante taxonomía clásica como hibridos. El grupo 2, si bien estaba constituido por $P$. chilensis, fue el que le siguió en orden decreciente $(\bar{H}=0,28)$. 


\section{Cuadro $\mathrm{N}^{\circ} 5$ \\ PROPORCIÓN DE HETEROCIGOTAS $\left(\mathrm{H}_{0}\right)$, HETEROCIGOSIS CONDICIONAL $\left(\mathrm{H}_{\mathrm{C}}\right)$ Y PROPORCIÓN MEDIA DE HETEROCIGOTAS $\left(\mathrm{H}_{M}\right)$}

\begin{tabular}{|c|l|l|l|l|l|l|l|l|l|l|}
\hline & ADH-A & ADH-B & GOT-A & GOT-B & 6 PGDH-A & 6 PGDH-B & MNR & PGI & SKDH & $H_{m}$ \\
\hline Grupo 1 & & & & & & & & & & \\
\hline$H_{0}$ & 0,086 & 0,000 & 0,262 & 0,312 & 0,199 & 0,333 & 0,498 & 0,158 & 0,328 & 0,24 \\
\hline$H_{c}$ & 0,543 & 0,000 & 0,262 & 0,446 & 0,381 & 0,744 & 0,530 & 0,588 & 0,328 & \\
\hline Grupo 2 & & & & & & & & & & \\
\hline$H_{0}$ & 0,246 & 0,000 & 0,224 & 0,394 & 0,306 & 0,268 & 0,375 & 0,235 & 0,451 & 0,28 \\
\hline$H_{c}$ & 0,879 & 0,000 & 0,224 & 0,439 & 0,520 & 0,311 & 0,375 & 0,964 & 0,493 & \\
\hline Grupo 3 & & & & & & & & & \\
\hline$H_{0}$ & 0,333 & 0,000 & 0,372 & 0,349 & 0,368 & 0,184 & 0,420 & 0,490 & 0,548 & $\mathbf{0 , 3 4}$ \\
\hline$H_{c}$ & 0,520 & 0,000 & 0,556 & 0,349 & 0,558 & 0,346 & 0,420 & 0,769 & 0,548 & \\
\hline Grupo 4 & & & & & & & & & \\
\hline$H_{0}$ & 0,500 & 0,000 & 0,317 & 0,410 & 0,308 & 0,167 & 0,382 & 0,345 & 0,432 & $\mathbf{0 , 3 2}$ \\
\hline$H_{c}$ & 0,602 & 0,000 & 0,379 & 0,421 & 0,510 & 0,538 & 0,579 & 0,746 & 0,439 & \\
\hline Grupo 5 & & & & & & & & & \\
\hline$H_{0}$ & 0,238 & 0,000 & 0,265 & 0,350 & 0,099 & 0,250 & 0,158 & 0,423 & 0,207 & 0,22 \\
\hline$H_{c}$ & 0,586 & 0,000 & 0,284 & 0,350 & 0,105 & 0,429 & 0,469 & 0,759 & 0,207 & \\
\hline
\end{tabular}

Diversos autores han determinado la proporción media de heterocigotas para distintas especies del género Prosopis. Asi, Verga (1995), en el mismo trabajo mencionado previamente, halló que los grupos de híbridos fueron los más variables, situación similar a la ocurrida en este estudio; mientras que $P$. chilensis $(\bar{H}=0,230)$ fue el menos variable y $P$. flexuosa $(\bar{H}=0,290)$, un algarrobo negro, se situó en valores cercanos a los grupos intermedios $(\bar{H}=0,330)$.

Con posterioridad, Saidman et al. (1998), presentaron una recopilación sobre investigaciones realizadas hasta ese momento para varias poblaciones y especies de Prosopis con los siguientes valores promedios para $P$. alba, $P$. nigra y $P$. flexuosa: $\bar{H}=0,184, \bar{H}=0,226$ y $\bar{H}=0,255$ respectivamente.

El grupo 5 representado por $P$. nigra fue el menos variable en esta investigación y presentó valores $(\bar{H}=0,220)$ que se acercan a los obtenidos por Saidman et al. $(1993 ; 1998)$. Para $P$. alba y $P$. flexuosa Saidman (1993) obtuvo valores de $\bar{H}$ iguales a 0,17 y 0,23 respectivamente, estudiando 25 loci. El mismo nivel encontraron para $P$. alba Montoya (1994) y Ferreyra (2000) analizando 24 loci.

Julio (2000) evaluando 17 loci en 12 poblaciones de $P$. chilensis encontró una $\bar{H}=$ 0,247 con una variación entre $0,195-0,296$, mientras que Ferreyra (2000) halló los siguientes valores de diversidad genética: $P$. flexuosa $(\bar{H}=0,258)$ y $P$. nigra $(\bar{H}=0,271)$ para 24 loci.

Por otra parte, el grupo 1 conformado por $P$. alba principalmente, presentó una proporción media de heterocigotas $(\bar{H}=0,240)$ inferior a la encontrada para $P$. chilensis $(\bar{H}=0,280)$, pero muy por encima de los valores registrados por los autores mencionados. 
El número efectivo de alelos $\left(A_{e s}\right)$ presentado por Hamrick et al. (1991) dentro de especies $\left(A_{e s=} 0,177\right)$ es comparable con la diversidad $v$ que tuvo estos valores: 1,$610 ; 1,705$; 1,$864 ; 1,738$ y 1,798 para los grupos morfológicos $1,2,3,4$ y 5 respectivamente.

La alta diversidad genética encontrada en todos los grupos morfológicos puede deberse a que se trata de unidades taxonómicas (especies o semiespecies leñosas) que poseen un amplio rango de distribución geográfica, sistema de cruzamiento abierto, con dispersión de la semilla por ingesta animal como lo señaló Hamrick et al. (1991). Por otra parte, la mayor diversidad encontrada en los grupos morfológicos 3 y 4 es congruente con la característica de grupos intermedios.

De aquí se desprende que un análisis basado únicamente en la taxonomia clásica hubiera llevado a una conclusión falsa. Los grupos 3 y 4 , analizados mediante la taxonomía numérica aparecen como las áreas más ricas en formas, coincidiendo con la observación del análisis genético en cuanto a su mayor diversidad, mientras que el grupo 1, el menos diverso morfológicamente, también lo es desde el punto de vista genético.

El grupo morfológico 2, representado por $P$. chilensis, no se presentó en la zona 1 , sin embargo la clasificación realizada por la taxonomía clásica, cita que está presente. Esta aparente contradicción surge del hecho que el taxón clasificado como $P$. chilensis entra en esta zona en el grupo morfológico 1, por lo que, según el análisis numérico, no se trataría de esta especie, sino de individuos cuya morfología estaría dentro de la variación de $P$. alba.

El predominio de híbridos maduros y jóvenes hace suponer que las perturbaciones en esta zona han comenzado hace tiempo y aún continúan. De todos los híbridos presentes en esta región el más representativo seria $P$. chilensis $\times P$. alba que aparece con tres grupos etarios.

Como se dijo anteriormente las zonas más diversas son la 3 y 4 y la menos diversa la 1. Todas estas poblaciones presentan la contradicción de que si se las compara con la cantidad de taxones presentes según la taxonomía clásica, parecería que la diversidad fuera diferente. Ahora si se toma la proporción de los grupos morfológicos distribuidos por zonas en vez de tener en cuenta la clasificación taxonómica clásica como se indica más arriba, se puede explicar mejor lo que acontece.

La participación de los grupos morfológicos intermedios (grupos 3 y 4 ) en cada zona es diferente, asi se tiene que los mayores porcentajes se encuentran en las zonas 3 y 4 ( $29 \%$ y $23 \%)$, mientras que los menores están en la zona 1 y $2(20 \%$ y $23 \%)$. En general los valores de diversidad genética también se ordenan en este sentido.

Este análisis apoya una vez más el concepto de que la taxonomía clásica puede llevar a confusiones al tratar de comprender las discontinuidades morfológicas presentes en el corredor bajo estudio y confirma la hipótesis de estas discontinuidades pueden ser ordenadas en 5 grupos morfológicos con una base genética definida. 


\section{Relaciones entre las Distancias Morfológicas y Genéticas}

Se calculó los coeficientes de correlación entre las matrices de distancia morfológica $\left(d_{m}\right)$ y genética $\left(d_{o}\right)$ correspondientes a los 5 grupos morfológicos. Estos valores indican un alto grado de correlación significativo entre las distancias morfológicas y genéticas de los grupos morfológicos (cuadro $\mathrm{N}^{\circ} 6$ ).

Existe una alta correlación significativa $(P<0,05)$ entre las matrices de distancias del pool de genes y de los caracteres morfológicos. Asimismo algunos de los caracteres que tienen correlación significativa con el pool de genes también la tienen con las enzimas ADH-A y MNR. Asi, de los 19 caracteres morfológicos estudiados, 12 presentan coeficientes de correlación (R) altos con el pool de genes y ADH-A, mientras que 11 lo hacen la MNR.

La alta correlación $\left(R^{2}=0,592\right)$ existente entre las distancias morfológicas y genéticas de los grupos morfológicos confirman la base genética de estos grupos, obtenidas a través de taxonomía numérica.

De igual manera Verga (1995) ordenó el aparente continuo entre Prosopis flexuosa, grupos intermedios y P. chilensis en el Chaco Árido al identificar de que cada uno respondía a un grupo morfológico determinado con base genética y con una alta correlación entre las matrices de distancia morfológica con la del pool de genes $\left(R^{2}=0,831\right)$ y con el locus $A D H-A$ $\left(R^{2}=0,874\right)$.

\section{Cuadro $\mathrm{N}^{\circ} 6$}

CORRELACIÓN ENTRE LAS MATRICES DE DISTANCIA MORFOLÓGICA Y GENÉTICA

\begin{tabular}{|c|c|c|c|c|}
\hline $\begin{array}{c}\text { Grupos } \\
\text { Morfológicos } \\
\text { Comparados }\end{array}$ & $\begin{array}{c}\text { Distancia Morfológica } \\
\left(\boldsymbol{d}_{\boldsymbol{m}}\right)\end{array}$ & $\begin{array}{c}\text { Distancia del Pool } \\
\mathbf{d e} \text { Genes } \\
\left(\boldsymbol{d}_{\mathrm{o}}\right)\end{array}$ & ADH-A & MNR \\
\hline $1 \times 2$ & 0,3786 & 0,111 & 0,074 & 0,187 \\
\hline $1 \times 3$ & 0,4204 & 0,154 & 0,225 & 0,171 \\
\hline $1 \times 4$ & 0,5221 & 0,139 & 0,347 & 0,140 \\
\hline $1 \times 5$ & 0,6643 & 0,270 & 0,742 & 0,313 \\
\hline $2 \times 3$ & 0,4228 & 0,167 & 0,181 & 0,217 \\
\hline $2 \times 4$ & 0,5747 & 0,147 & 0,276 & 0,323 \\
\hline $2 \times 5$ & 0,6765 & 0,243 & 0,682 & 0,498 \\
\hline $3 \times 4$ & 0,4913 & 0,110 & 0,095 & 0,224 \\
\hline $3 \times 5$ & 0,6026 & 0,229 & 0,501 & 0,386 \\
\hline $4 \times 5$ & 0,4710 & 0,193 & 0,406 & 0,175 \\
\hline$R 2$ & & $\mathbf{0 , 5 9 2}$ & $\mathbf{0 , 7 6 4}$ & $\mathbf{0 , 6 6 1}$ \\
\hline $\mathrm{R}$ & & $\mathbf{0 , 7 6 9}$ & $\mathbf{0 , 8 7 4}$ & $\mathbf{0 , 8 1 2}$ \\
\hline
\end{tabular}

Referencia: en negrita nivel de significancia $P<0,05$. 


\section{Los Grupos Morfológicos-Genéticos}

En este trabajo, tres grupos morfológicos-genéticos podrían ser considerados "buenas" especies (grupo $1=P$. alba, grupo $2=P$. chilensis y grupo $5=P$. nigra). De estas la de mayor heterosigosis fue $P$. chilensis, y la menor $P$. nigra, sin embargo desde el punto de vista de los indices de diversidad ambas especies revelan valores similares.

El grupo morfólogico-genético 1 que, desde la taxonomia clásica estaria formada por $P$. alba, P.chilensis con foliolulos menores, $P$. chilensis e híbridos entre ambas, fue el grupo de menor diversidad y heterosis. En el análisis de reasignación de grupos de los caracteres morfológicos de las madres tuvo una baja tasa de error y la mayoria de los individuos que lo conformaban pudieron ser reasignados a este grupo morfológico. Esto estaría indicando que se trata de una sola especie $(P$. alba).

En cuanto a los caracteres morfológicos de la descendencia de este grupo morfológico, segregaron al ser reasignados, por lo que se puede sospechar que este grupo puede cruzarse con individuos de otros grupos morfológicos.

Los grupos morfológicos 3 y 4 confirman su condición de intermedios, pues fueron los que mayor diversidad y variabilidad presentaron. Estos datos coinciden con las observaciones realizadas por Verga (1995) en cuanto a que el complejo estaria conformado por especies "inespecificas" que tendrian la capacidad de contener alta variabilidad genética, proveniente de especies más "especificas" adaptadas a nichos más estrechos.

A partir de la recombinación en áreas de contacto secundario entre las distintas "especies" del complejo, donde surgen procesos de introgresión, existiria la posibilidad de que se generen formas nuevas, que si encuentran nichos adecuados y posibilidades de aislamiento genético (pre o postcigótico) de las "especies parentales" podrian constituirse, luego de un tiempo de diferenciación, en entidades identificables desde el punto de vista taxonómico, genético y ecofisiológico.

\section{CONCLUSIONES}

La gran diversidad morfológica, existente en poblaciones del género Prosopis del Chaco Semiárido del Norte de Córdoba y Sudeste de Santiago del Estero, fue ordenada en 5 grupos morfológicos con una base genética característica para cada grupo. Los estudios morfológicos de la descendencia confirmaron la existencia de los grupos morfológicos-genéticos definidos. 


\section{REFERENCIAS}

Anderson E., 1949. Introgressive hybridization. Wiley, Nueva York.

Bessega, C., 1997. Estudios isoenzimáticos en especies Americanas del Género Prosopis (Leguminosae). Tesis de Maestría. FCE y N. Universidad de Buenos Aires. Buenos Aires).

Burghardt, A., 1992. Prosopis L. Caracterización electroforètica de sus especies Tesis Doctoral Departamento de Ciencias Biológicas.U.B.A

Burghardt, A.y Palacios, R. A., 1981. Caracterización electroforètica de algunas especies del género Prosopis (Leguminosae). XII Congreso Argentino de Genética :11.

Burghardt, A. D., Brizuela, M. M. y Palacios, R. A., 2000. Variabilidad en plántulas de algunas especies de Prosopis (Fabaceae). En busca de descriptores morfológicos. Multequina 9:23-33.

Burkart, A., 1976. A monograph of the genus Prosopis (Leguminosae subfam. Mimosoideae) (Part 1 and 2). Catalogue of the recognized species of Prosopis. Journal of the Arnold Arboretum. 57:219-249 y 450-525.

De Fina, A., 1976. Datos agroclimáticos de la República Argentina. IDIA 337-342:57-186. Dirección de Recursoso Forestales Nativos.1992 Desarrollo Sustentable o Deforestación. Plan Forestal Argentino. 55 pp.

FAO-PNUMA,1997. Especies arbóreas y arbustivas para las zonas áridas y semiáridas de América Latina. Serie: Zonas Áridas y Semiáridas $N^{\circ} 12$. 347pp.

Ferreira, L. I., 2000. Estudio de la variabilidad y la diferenciación genética por medio de técnicas de Isoenzimas y RAPD en poblaciones naturales de especies e híbridos de Género Prosopis (Leguminosae). Tesis para optar al grado de Doctor en Ciencias Biológicas. Universidad de Buenos Aires. Facultad de Ciencias Exactas y Naturales. 200 pp.

Grant, V., 1981. Plant speciation. Columbia Univ.Press. New York.

Gregorius, H. R., 1974. Genetischer Abstand zwischen Populationen. I. Zur Konzeption der genetischen Abstandsmessung. Silvae Genetica.23:1-3.

Hamrick, J. L., Godt, M.J. W. y Sherman-Broyles, S. L.,1991. Factors influencing levels of genetic diversity in woody plant species. Review Paper. New Forest 6:94-124. Kluver Academic Publisher.

Hunziker, J. H., Poggio, L., Naranjo, C. A. y Palacios, R. A., 1975. Cytogenetics of some species and natural hybrids in Prosopis (Leguminosae). Canadian Journal of Genetics and Cytology. 17:253:262.

Hunziker, J., Naranjo, C., Palácios, R. A. and Poggio, L.1977. Chromosomal cytology and hybridization. En: Simpson, B. Mesquite. Its biology in two desert ecosystems. US/IBP. Sweries $4 \mathrm{Ch}$. 3. Patterns of variation. Dowden, Hutchinson and Ross, Inc.: 56-59.

Hunziker, J. H., Saidman, B. O., Naranjo, C. A., Palacios, R. A., Poggio, L. y Burghardt, A. D.1986. Hybridization and genetic variation of Argentine species of Prosopis (Leguminosae, Mimosoidae). Forest Ecology and Management 16:301-315.

Joseau, M. J., Verga, A. R. y Díaz, M. del Pilar, 2004. Los recursos genéticos de Prosopis en el corredor que une el Chaco árido con el semiárido entre las Provincias de Córdoba y Santiago del 
Estero. Genética y Mejoramiento. Revista IDIA XXI:207-211. Revista de información sobre investigación y desarrollo agropecuario. Forestales. INTA.

Joseau, M. J. y Castro Schule, F. F., 2005. Caracterización del género Prosopis. En: Conservación de recursos forestales nativos en Argentina. El Banco Nacional de Germoplasma de Prosopis.

Verzino, G. E. y M. J. Joseau (Eds). Córdoba, Argentina. VIII:81-88 pp.

Julio, N. B., 2000. Estudios alozimicos sobre variabilidad, estructura y diferenciación genética en Prosopis chilensis (Leguminosae. Mimosoideae) y especies relacionadas. Tesis de doctorado en Ciencias Biológicas. Universidad Nacional de Córdoba. Facultad de Ciencias Exactas, Físicas y Naturales.153 pp.

Mantován, N. G., 2000 a. Variabilidad intraespecifica de los patrones fenológicos de Prosopis flexuosa en la provincia fitogeográfica del Monte. Reunión Nacional del Algarrobo. Mendoza, p.30.

Mantován, N. G., 2000 b. Diferenciación morfológica entre poblaciones de Prosopis flexuosa D.C. en la provincia fitogeográfica del Monte, Argentina.III Reunión Nacional de la Asociación Argentina de Prosopis. Mendoza. Argentina.14-17 de Noviembre de 2000. p 38.

Montoya, S., Saidman, B. O., Vilardi, J. C. y Bessega, C., 1994. Diferenciación y flujo genético entre especies de la Sección Algarobia. Género Prosopis (Leguminosae). Actas del XXIV Congreso de la Sociedad Argentina de Genética. La Plata. 17.

Morillo, J., Crudeli, N. y Sarraceno, M.,1971. Los vinales de Formosa (Rep. Argentina). La colonizadora leñosa Prosopis ruscifolia Gris. Serie fitogeográfica 11. INTA.

Naranjo, C. A. y Enus Zeiger, S., 1983. Cromatografia de fenoles y morfologia en especies e hibridos de Prosopis de la Pampa. XIX Jornadas Arg. de Botánica:32.

Naranjo, C. A., Poggio, L. y Enus Zeiger, S., 1984. Phenol chromatography, morphology and cytogenetics in three species and natural hybrids of Prosopis (Leguminosae, Mimosoidae). PI. Syst. Evol.,144:257-276.

Palacios, R. A. y L. D. Bravo, 1981. Hibridación natural en Prosopis (Leguminosae) en la región chaqueña argentina. Evidencias morfológicas y cromatográficas. Darwiniana, 23:3-35

Saidman, B. O., 1985. Estudio de la variación alozímica en el género Prosopis. Tesis doctoral. Fac. Cs. Exactas y Nat. , Universidad de Buenos Aires, Buenos Aires.

Saidman, B. O., 1986. Isoenzymatic studies of alcohol dehydrogenase and glutamate oxalacetate transaminase in four South American specis of

Prosopis and their natural hybrids. Silvae Genetica 35:3-10.

Saidman, B. O., 1988. La electroforesis de isoenzimas para la medición de la variabilidad genética en especies de Prosopis. In: Prosopis en Argentina. Documento preliminar elaborado para el I Taller Internacional sobre Recurso Genético y Conservación de Germoplasma en Prosopis. Fac. de Cs. Agropecuiarias (U.N.C.)FAO- PIRB. P 107-118.

Saidman, B. O., 1993. Las isoenzimas en el estudio de la variación genética y las afinidades entre especies de Prosopis. Bol. Genét. Inst. Fitotéc. Castelar 16:25-37.

Saidman, B. O. y Vilardi, J. C., 1987. Analysis of the genetic similarities among seven species of Prosopis (Leguminosae: Mimosoideae) Theoret. Appl. Genet. 75:109-116. 
Saidman, B. O. and Vilardi, J.C.,1993. Genetic variability and germoplasm conservation in the genus Prosopis. In: Nursery technology of forest tree species of arid and semiarid regions (ed S. Puri), Winrock- Oxford \& IBH Publishing Co. PVT. Ltd., New Delhi, Bombay, Calcuta, pp.187-198.

Saidman, B. O., Montoya, S., Vilardi, J. C. and Poggio, L., 1997 a. Genetic variability and ploidy level in species of Prosopis. Bol. Soc. Argent. Bot. 32:217-225.

Saidman, B. O., Vilardi J. C., Montoya, S., Dieguez, M. J. and Hopp, H. E., 1998. Molecular markers: a tool for the understanding of the relationships among sepecies of Prosopis (Leguminosae, Mimosoidae). In: Tree Improvement: Applied Research and Technology Transfer (eds. S. PURI), Science Publishers Inc. U.S.A.21:311-324.

Saidman, O. B., Bessega , C. F., Ferreira, L. I., Julio, N. and Vilardi, J. C. 2000. The use of genetic markers to assess population structure and relationships among species of the genus Prosopis (Leguminosae). Bol. Soc. Argent. Bot. (3-4):315-324.

Solbrig, O. T., Bawa, K., Carman, N. J., Hunziker, J. H., Naranjo, C. A, Palacios, R. A., Poggio, L. y Simpson, B. B. 1977. Patterns of variation. Mesquite, Its Biology in Two Desert Ecosystems. pp44-60. (Ed.) B.B Simpson. Dowden, Hutchinson and Ross, Stroudsburg. Pennsylvania, USA.

Verga, A. R.,1995. Genetic study of Prosopis chilensis y Prosopis flexuosa (Mimosaceae) in the dry Chaco of Argentina.Tesis Doctoral. Göttingen Researh Notes in Forest Genetics. Abteilung für Forstgenetik und Forstpflanzensüchtung der Universität Göttingen.

Verga, A. R., 2000 a. Algarrobos como especies para forestación. una estrategia de mejoramiento. SAGPyA Forestal $n^{\circ} 16: 12-18$. Septiembre 2000. $1^{\circ}$ parte.

Verga, A. R. 2000 b. Algarrobos como especies para forestación. una estrategia de mejoramiento. SAGPyA Forestal n¹7:2-9 Diciembre 2000. Primera parte.

Verga, A., Carranza, C., Ledesma, M., Joseau, J., Córdoba, A., Montura, M., López Lauenstein, D., Recalde, D., Orionte, E., Tomalino, L., Mendoza, S. y Vega, R., 2003. Biodiversidad y resguardo de los recursos genéticos: conservación, mejoramiento genético y silvicultura del algarrobo en el Chaco Árido Argentino. $2^{\circ}$ Congreso de Nacional sobre Manejo de Pastizales Naturales. $6^{\circ}$ Jorn Reg. IV Reunión de la Asoc. Argentina de Prosopis. San Cristobal. Sta Fe. Argentina. 8-10 de Oct. 2003. Volumen 1. Resúmenes. $66 \mathrm{p}$.

Verga, A., Cony, M., Joseau, J., López, D. y Córdoba, A., 2004. Estudio Genético preliminar en cuatro zonas de Algarrobo Dulce y Caldén. ( $P$. flexuosa y $P$. caldenia ) en Mendoza y San Luis. Sin publicar.

Verga, A. and Gregorius, H. R., (Unpublish). Genetic consistency of morphological differentiation in the Prosopis chilensis-P. flexuosa complex. 
